\title{
Some properties of solutions for a nonlinear integral system
}

\author{
Xiaoying Wang ${ }^{1}$, Junjie $\mathrm{Li}^{2}$ and Jiankai $\mathrm{Xu}^{3^{*}}$
}

\section{*Correspondence:}

jiankaixu@126.com

${ }^{3}$ College of Sciences, Hunan

Agriculture University, Hunan,

410128, China

Full list of author information is

available at the end of the article
2015 Wang et al. This article is distributed under the terms of the Creative Commons Attribution 4.0 International License (http://creativecommons.org/licenses/by/4.0/), which permits unrestricted use, distribution, and reproduction in any medium, provided you give appropriate credit to the original author(s) and the source, provide a link to the Creative Commons license, and indicate if changes were made.

\begin{abstract}
In this paper, a nonlinear integral system is considered in critical space. Some important properties of positive solutions such as symmetry, monotonicity, integrability, and asymptotic behaviors, are obtained. Moreover, by comparison and analysis, we discover that those properties are an important tool to characterize the tightness of the system.
\end{abstract}

Keywords: integral system; regularity lifting lemma; weighted Hardy-Littlewood-Sobolev inequality

\section{Introduction}

In this paper, we consider the following nonlinear integral system involving the weighted Riesz potentials:

$$
\left\{\begin{array}{l}
u(x)=\int_{\mathbb{R}^{n}} \frac{\nu^{p}(y) u^{q}(y) w^{r}(y)}{|x-y|^{\lambda}} \frac{1}{|y|^{\beta}} d y, \\
v(x)=\int_{\mathbb{R}^{n}} \frac{v^{r}(y) u^{p}(y) w^{q}(y)}{|x-y|^{\lambda}} \frac{1}{|y|^{\beta}} d y, \\
w(x)=\int_{\mathbb{R}^{n}} \frac{v^{q}(y) u^{r}(y) w^{p}(y)}{|x-y|^{\lambda}} \frac{1}{|y|^{\beta}} d y,
\end{array}\right.
$$

where $0<\lambda<n, 0<\beta+\lambda<n$, and $p, q, r \geq 1$ satisfying $p+q+r=(2 n-\lambda-2 \beta) / \lambda$.

Recently, there has been tremendous interest in studying integral systems. Because the integral equation(s) not only formulate abstractly many laws and relations in science, engineering, economics, and other fields of applied science, but also they provide a special technique to investigate the global properties of the corresponding differential equation(s) due to the fact that the integral equation(s), under certain integrability conditions, is (are) equivalent to differential equation(s). We recall some related background and investigations as follows.

As $u(x)=v(x)=w(x)$ and $0<\beta<n-\lambda, p+q+r=2(n-\beta) / \lambda-1$, the system (1.1) can be reduced to the following single equation:

$$
u(x)=\int_{\mathbb{R}^{n}} \frac{u^{p+q+r}(y)}{|x-y|^{\lambda}|y|^{\beta}} d y .
$$

Lu and Zhu in [1] obtained some regularity results and showed that every positive solution $u(x)$ is radially symmetric about the origin and strictly decreasing in $L^{2 n / \lambda}\left(\mathbb{R}^{n}\right)$. Subsequently, under the same conditions, Lei in [2] showed that for $0<p+q+r \leq(n-\beta) / \lambda$, 
equation (1.2) has no positive solution and that for $p+q+r>(n-\beta) / \lambda$, all of positive solution of (1.2), $u \in L^{2 n / \lambda}\left(\mathbb{R}^{n}\right)$ is bounded and decays fast with rate $|x|^{-\lambda}$.

When $\beta=0, \lambda=n-2$ and $w(x)=v(x)$, the system (1.1) can be rewritten as

$$
\left\{\begin{array}{l}
u(x)=\int_{\mathbb{R}^{n}} \frac{v^{q_{1}(y) u^{p^{1}}(y)}}{|x-y|^{\lambda}} d y, \\
v(x)=\int_{\mathbb{R}^{n}} \frac{u^{p_{1}(y) \nu^{1}(y)}}{|x-y|^{\lambda}} d y .
\end{array}\right.
$$

The equations (1.3), under some integrability condition, is equivalent to the following differential system:

$$
\begin{cases}-\Delta u(x)=v^{q_{1}}(x) u^{p_{1}}(x) & \text { in } \mathbb{R}^{n}, \\ -\Delta v(x)=u^{p_{1}}(x) v^{q_{1}}(x) & \text { in } \mathbb{R}^{n},\end{cases}
$$

which are closely related to stationary Schrödinger system with critical exponents for the Bose-Einstein condensate. Li and Ma in [3] showed that for $n \geq 3,1 \leq p_{1}, q_{1} \leq$ $(n+2) /(n-2)$, and $p_{1}+q_{1}=(n+2) /(n-2)$, any positive solution pair $(u, v)$ of system (1.4) in $L^{2 n /(n-2)}\left(\mathbb{R}^{n}\right) \times L^{2 n /(n-2)}\left(\mathbb{R}^{n}\right)$ is radially symmetric and unique.

Later on, Zhao and Lei in [4] considered the following weighted nonlinear system:

$$
\left\{\begin{array}{l}
u(x)=\int_{\mathbb{R}^{n}} \frac{v^{q_{1}(y) u^{p_{1}(y)}}}{|x-y|^{\lambda}|y|^{\beta}} d y, \\
v(x)=\int_{\mathbb{R}^{n}} \frac{u^{p^{1}(y) \nu^{p_{1}}(y)}}{|x-y|^{\lambda}|y|^{\beta}} d y .
\end{array}\right.
$$

The authors in [4] showed that as $\lambda+\beta<n, 0 \leq \beta<\lambda$ and $p_{1}+q_{1}=(2 n-\lambda-\beta) /(\lambda+\beta)$, any positive solution pair $(u, v)$ of equation (1.5) is in $L^{2 n /(\lambda+\beta)}\left(\mathbb{R}^{n}\right) \times L^{2 n /(\lambda+\beta)}\left(\mathbb{R}^{n}\right)$. Additionally, if $(n-\lambda)(\lambda+\beta) \geq 2 n \beta$, then the positive solution pair $(u, v)$ of system (1.5) is bounded and satisfies

$$
\lim _{|x| \rightarrow \infty}|x|^{\lambda} u(x)=\int_{\mathbb{R}^{n}} \frac{u^{q}(y) v^{p}(y)}{|y|^{\beta}} d y, \quad \lim _{|x| \rightarrow \infty}|x|^{\lambda} v(x)=\int_{\mathbb{R}^{n}} \frac{u^{p}(y) v^{q}(y)}{|y|^{\beta}} d y .
$$

An analogous integral system of (1.1) is

$$
\left\{\begin{array}{l}
u(x)=\frac{1}{|x|^{\alpha}} \int_{\mathbb{R}^{n}} \frac{v^{q}(y)}{|x-y|^{\lambda}|y|^{\beta}} d y, \\
v(x)=\frac{1}{|x|^{\beta}} \int_{\mathbb{R}^{n}} \frac{u^{p}(y)}{|x-y|^{\lambda}|y|^{\alpha}} d y .
\end{array}\right.
$$

It is closely related to the best constant in the weighted Hardy-Littlewood-Sobolev inequality.

Assume that $1<r, s<\infty, 0<\lambda<n, \alpha+\beta \geq 0,1 / r+1 / s+(\lambda+\alpha+\beta) / n=2$, and $1-1 / r-$ $\lambda / n \leq \alpha / n<1-1 / r$, the well-known weighted Hardy-Littlewood-Sobolev inequality states that

$$
\left.\left|\int_{\mathbb{R}^{n}} \int_{\mathbb{R}^{n}}\right| x\right|^{-\alpha} f(x) g(y)|x-y|^{-\lambda}|y|^{-\beta} d x d y \mid \leq C(\lambda, \alpha, \beta, n)\|f\|_{L^{r}\left(\mathbb{R}^{n}\right)}\|g\|_{L^{s}\left(\mathbb{R}^{n}\right)}
$$

for any $f \in L^{r}\left(\mathbb{R}^{n}\right)$ and $g \in L^{s}\left(\mathbb{R}^{n}\right)$. 
To find the best constant $C(\lambda, \alpha, \beta, n)$ in the above inequality, one can maximize the functional

$$
J(f, g)=\int_{\mathbb{R}^{n}} \int_{\mathbb{R}^{n}}|x|^{-\alpha} f(x) g(y)|x-y|^{-\lambda}|y|^{-\beta} d x d y,
$$

under the constraints

$$
\|f\|_{L^{r}\left(\mathbb{R}^{n}\right)}=\|g\|_{L^{s}\left(\mathbb{R}^{n}\right)}=1 .
$$

The corresponding Euler-Lagrange equations are the following integral system:

$$
\left\{\begin{array}{l}
\lambda_{1} r f^{r-1}(x)=\frac{1}{|x|^{\alpha}} \int_{\mathbb{R}^{n}} \frac{g(y)}{|x-y|^{\lambda}|y|^{\beta}} d y, \\
\lambda_{2} s g^{s-1}(x)=\frac{1}{|x|^{\beta}} \int_{\mathbb{R}^{n}} \frac{f(y)}{|x-y|^{\lambda}|y|^{\alpha}} d y .
\end{array}\right.
$$

Here $f, g \geq 0$, and $\lambda_{1} r=\lambda_{2} s=J(f, g)$. After a nonlinear transformation, i.e. $u=c_{1} f^{r-1}$, $v=c_{2} g^{s-1}$, the integral system (1.8) becomes (1.7). By the method of moving plane in integral form, Jin and Li in [5] showed that all of the positive solutions of (1.7) are radially symmetric. Subsequently, they used the regularity lifting lemma and showed, when $\alpha, \beta>0$, that $0<\lambda<n$ and $p, q>1$ satisfy

$$
\frac{1}{p+1}+\frac{1}{q+1}=\frac{\bar{\lambda}}{n}, \quad \frac{1}{p+1}-\frac{\lambda}{n}<\frac{\alpha}{n}<\frac{1}{p+1}
$$

Then

$$
u \in L^{r}\left(\mathbb{R}^{n}\right), \quad v \in L^{s}\left(\mathbb{R}^{n}\right),
$$

provided that, for $(q+1)(\lambda+2 \beta) \geq 2 n$,

$$
\frac{1}{r} \in\left(\frac{\max \{\alpha, \beta q+\bar{\lambda}-n\}}{n}, \frac{\lambda+\alpha}{n}\right), \quad \frac{1}{s} \in\left(\frac{\beta}{n}, \frac{\min \{\lambda+\beta, p(\lambda+\alpha)+\bar{\lambda}-n\}}{n}\right),
$$

and for $(q+1)(\lambda+2 \beta)<2 n$

$$
\frac{1}{r} \in\left(\frac{\alpha}{n}, \frac{\min \{\lambda+\alpha, q(\lambda+\beta)+\bar{\lambda}-n\}}{n}\right), \quad \frac{1}{s} \in\left(\frac{\max \{\beta, p \lambda+\bar{\lambda}-n\}}{n}, \frac{\beta+\lambda}{n}\right) .
$$

Furthermore, with the help of the integrability and symmetry of positive solution for (1.7), Li, Lim, Lei and Ma in [6-8] obtained the sharp asymptotic estimates as follows.

When $\alpha+\beta \geq 0$, the pair of solutions $(u, v)$ of $(1.7)$ have the following asymptotic behaviors at the origin:

$$
u(x) \simeq \frac{A_{0}}{|x|^{\alpha}}, \quad \text { if } \lambda+(q+1) \beta<n,
$$


and

$$
v(x) \bumpeq \begin{cases}\frac{A_{1}}{|x|^{\beta}}, & \text { if } \lambda+\alpha(p+1)<n, \\ \frac{A_{2}|\ln | x||}{|x|^{\beta}}, & \text { if } \lambda+\alpha(p+1)=n, \\ \frac{A_{3}}{|x|^{\alpha(p+1)+\beta+\lambda-n}}, & \text { if } \lambda+\alpha(p+1)>n .\end{cases}
$$

At the same time, $(u, v)$ at infinity admits the following asymptotic behaviors:

$$
u(x) \simeq \frac{B_{0}}{|x|^{\lambda+\alpha}}, \quad \text { if } \lambda q+\beta(q+1)>n,
$$

and

$$
v(x) \simeq \begin{cases}\frac{B_{1}}{|x|^{\beta+\lambda}}, & \text { if } \lambda p+\alpha(p+1)>n, \\ \frac{B_{2}|\ln | x||}{|x|^{\beta+\lambda}}, & \text { if } p+\alpha(p+1)=n, \\ \frac{B_{3}}{|x|^{(\alpha+\lambda)(p+1)+\beta-n}}, & \text { if } p+\alpha(p+1)<n .\end{cases}
$$

Here, we use the notation $w(x) \simeq C /|x|^{t}$ to denote that $\lim _{x \rightarrow 0}$ or $\infty|x|^{t} w(x)=C$ for a function $w(x)$, a real number $t$, and a non-zero real number $C$.

In this paper, we will study some important properties of (1.1), such as symmetry, monotonicity, integrability, and asymptotic behaviors. At the same time, by comparison and analysis, we observe that the related properties are important tools to characterize the tightness of the system. Specifically, our main results can be formulated as follows.

Theorem 1.1 Let $(u, v, w)$ be a pair of positive solutions of $(1.1)$ and $p+q+r=(2 n-\lambda-$ $2 \beta) / \lambda$. Assume that $(u(x), v(x), w(x)) \in L^{s}\left(\mathbb{R}^{n}\right) \times L^{s}\left(\mathbb{R}^{n}\right) \times L^{s}\left(\mathbb{R}^{n}\right)$ for $s=n(p+q+r-1) /(n-$ $\beta-\lambda)$. Then:

$\mathrm{R}_{1} .(u, v, w)$ is radially symmetric and monotone decreasing about the origin.

$\mathrm{R}_{2}$. $(u, v, w)$ admits the same integral interval:

$$
u(x), v(x), w(x) \in L^{\tau}\left(\mathbb{R}^{n}\right), \quad \forall \tau \in\left(\frac{n}{\lambda}, \infty\right]
$$

$\mathrm{R}_{3}$. We have the optimal decay estimates

$$
\begin{aligned}
& \lim _{|x| \rightarrow \infty}\left[|x|^{\lambda} u(x)\right]=\int_{\mathbb{R}^{n}} \frac{v^{p}(y) u^{q}(y) w^{r}(y)}{|y|^{\beta}} d y \\
& \lim _{|x| \rightarrow \infty}\left[|x|^{\lambda} v(x)\right]=\int_{\mathbb{R}^{n}} \frac{v^{r}(y) u^{p}(y) w^{q}(y)}{|y|^{\beta}} d y ; \\
& \lim _{|x| \rightarrow \infty}\left[|x|^{\lambda} w(x)\right]=\int_{\mathbb{R}^{n}} \frac{v^{q}(y) u^{r}(y) w^{p}(y)}{|y|^{\beta}} d y .
\end{aligned}
$$

Remark 1.2 Comparing Theorem 1.1 with the results of (1.7) in [5], the pair of solutions has the same radial symmetry and is decreasing about the origin. But the integrable intervals and asymptotic behaviors of solutions for the system (1.1) are different from those of (1.7). The different structure leads to a Kelvin transform used in [5-12] to obtain the decay estimate of (1.7), which is invalid for the system (1.1). Therefore, we have to look for 
a new way to obtain the asymptotic result. Precisely, the difference between systems (1.1) and (1.7) leads to the thoroughly different asymptotic behavior at infinity. Indeed, by (1.11) and (1.12), we have learned that the asymptotic behavior of $u$ and $v$ in (1.7) is completely different, however, for the system (1.1), we know from (1.14), (1.15), and (1.16) that the solution $(u, v, w)$ has the same decay estimate at infinity. This implies that the triplet $(u, v, w)$ in (1.1) is tighter than those of system (1.7).

The other significant difference between (1.1) and (1.7) is the corresponding optimal integrable intervals of solutions. From (1.13), we know that the triplet $(u, v, w)$ in (1.1) admits the same optimal integrable intervals. However, for the pair of solutions $(u, v)$ in system (1.7), the optimal integrable interval of $u$ is different from the one of $v$.

Remark 1.3 We remark that the integral system (1.5) considered in [4] can be regarded as the special cases of (1.1) with $v=w$ and $r=p$. At the same time, the system (1.5) including its reduced model (1.3) studied in [3], have similar radial symmetry, monotonicity, and asymptotic behavior to our system (1.1). However, the solution space $L^{s}\left(\mathbb{R}^{n}\right) \times L^{s}\left(\mathbb{R}^{n}\right) \times$ $L^{s}\left(\mathbb{R}^{n}\right)$ with $s=n(p+q+r-1) /(n-\lambda)=2 n / \lambda$ in our theorems is different from $L^{s}\left(\mathbb{R}^{n}\right) \times$ $L^{s}\left(\mathbb{R}^{n}\right)$ with $s=2 n /(\lambda+\beta)$ in [4] since the index $p+q+r=(2 n-\lambda-2 \beta) / \lambda$ in our paper is different from one $p+q=(2 n-\lambda-\beta) /(\lambda+\beta)$ in [4]. Moreover, the method for the upper bound estimate of integrable interval to system (1.1) in our paper is completely different from the one in [4]. By contrast, our technique is more simple than the one used in [4].

The rest of this paper is organized as follows. In Section 2, we will consider $R_{1}$ and the proofs of $R_{2}$ will be given in Section 3. Finally, we will build up the sharp asymptotic estimates of (1.1) in Section 4.

Throughout this paper, we always use the letter $C$ to denote positive constants that may vary at each occurrence but are independent of the essential variables.

\section{Radial symmetry}

To obtain our results, in this section, we will use the method of moving plane in the integral forms recently introduced by Chen et al. in [13] and prove the radial symmetry and monotonicity of positive solutions of system (1.1). First of all, we introduce some necessary lemma.

For a given real number $\mu \in \mathbb{R}$, define

$$
\Sigma_{\mu} \triangleq\left\{x=\left(x_{1}, \ldots, x_{n}\right) \in \mathbb{R}^{n}: x_{1} \geq \mu\right\}
$$

and let $x^{\mu} \triangleq\left(2 \mu-x_{1}, x_{2}, \ldots, x_{n}\right), u_{\mu}(x) \triangleq u\left(x^{\mu}\right), v_{\mu}(x) \triangleq v\left(x^{\mu}\right)$, and $w_{\mu}(x) \triangleq w\left(x^{\mu}\right)$.

Lemma 2.1 Let $(u(x), v(x), w(x))$ be a solution of system (1.1), then

$$
\begin{aligned}
& u_{\mu}(x)-u(x) \\
& =\int_{\Sigma_{\mu}}\left\{\left(\left[\frac{1}{|x-y|^{\lambda}}-\frac{1}{\left|x^{\mu}-y\right|^{\lambda}}\right] \frac{1}{\left|y^{\mu}\right|^{\beta}}\right)\left(v_{\mu}^{p} u_{\mu}^{q} w_{\mu}^{r}(y)-v^{p} u^{q} w^{r}\right)\right. \\
& \left.\quad+\left(\frac{1}{\left|x^{\mu}-y\right|^{\lambda}}-\frac{1}{|x-y|^{\lambda}}\right)\left(\frac{1}{|y|^{\beta}}-\frac{1}{\left|y^{\mu}\right|^{\beta}}\right) v^{p} u^{q} w^{r}\right\} d y \\
& \triangleq
\end{aligned}
$$




$$
\begin{aligned}
& v_{\mu}(x)-v(x) \\
& =\int_{\Sigma_{\mu}}\left\{\left(\left[\frac{1}{|x-y|^{\lambda}}-\frac{1}{\left|x^{\mu}-y\right|^{\lambda}}\right] \frac{1}{\left|y^{\mu}\right|^{\beta}}\right)\left(v_{\mu}^{r} u_{\mu}^{p} w_{\mu}^{q}(y)-v^{r} u^{p} w^{q}\right)\right. \\
& \left.\quad+\left(\frac{1}{\left|x^{\mu}-y\right|^{\lambda}}-\frac{1}{|x-y|^{\lambda}}\right)\left(\frac{1}{|y|^{\beta}}-\frac{1}{\left|y^{\mu}\right|^{\beta}}\right) v^{r} u^{p} w^{q}\right\} d y \\
& \triangleq B_{1}(x)+B_{2}(x) ;
\end{aligned}
$$

and

$$
\begin{aligned}
& w_{\mu}(x)-w(x) \\
& =\int_{\Sigma_{\mu}}\left\{\left(\left[\frac{1}{|x-y|^{\lambda}}-\frac{1}{\left|x^{\mu}-y\right|^{\lambda}}\right] \frac{1}{\left|y^{\mu}\right|^{\beta}}\right)\left(v_{\mu}^{q} u_{\mu}^{r} w_{\mu}^{p}(y)-v^{q} u^{r} w^{p}\right)\right. \\
& \left.\quad+\left(\frac{1}{\left|x^{\mu}-y\right|^{\lambda}}-\frac{1}{|x-y|^{\lambda}}\right)\left(\frac{1}{|y|^{\beta}}-\frac{1}{\left|y^{\mu}\right|^{\beta}}\right) v^{q} u^{r} w^{p}(y)\right\} d y \\
& \triangleq C_{1}(x)+C_{2}(x) .
\end{aligned}
$$

Proof By a direct calculation, it is easy to check that

$$
u(x)=\int_{\Sigma_{\mu}} \frac{v^{p} u^{q} w^{r}}{|x-y|^{\lambda}|y|^{\beta}} d y+\int_{\Sigma_{\mu}} \frac{1}{\left|x^{\mu}-y\right|^{\lambda}} \frac{v_{\mu}^{p} u_{\mu}^{q} w_{\mu}^{r}(y)}{\left|y^{\mu}\right|^{\beta}} d y
$$

and

$$
u_{\mu}(x)=\int_{\Sigma_{\mu}} \frac{v^{p} u^{q} w^{r}}{\left|x^{\mu}-y\right|^{\lambda}|y|^{\beta}} d y+\int_{\Sigma_{\mu}} \frac{1}{|x-y|^{\lambda}} \frac{v_{\mu}^{p} u_{\mu}^{q} w_{\mu}^{r}(y)}{\left|y^{\mu}\right|^{\beta}} d y
$$

(2.1) is a direct result of the above equations. Similarly, we get (2.2) and (2.3). This completes the proof of Lemma 2.1.

Proof of $\mathrm{R}_{1}$ Now, we turn to the first part of Theorem 1.1. The proof is made up of two steps. In Step 1, we compare the values of $u(x)$ with $u_{\mu}(x), v(x)$ with $v_{\mu}(x)$ and $w(x)$ with $w_{\mu}(x)$ on $\Sigma_{\mu}$, respectively, and show that for sufficiently negative $\mu<0$, we have

$$
u(x) \geq u_{\mu}(x), \quad v(x) \geq v_{\mu}(x) \quad \text { and } \quad w(x) \geq w_{\mu}(x) \quad \forall x \in \Sigma_{\mu}-\{0\} .
$$

In Step 2, we continuously move the plane $x_{1}=\mu$ along the $x_{1}$ direction from near negative infinity to the right as long as (2.4) holds. By moving this plane in this way, we finally show that the plane will stop at the origin. Next we turn our attention to Step 1.

Step 1: Since $A_{2}(x)<0$ and $\left|y^{\mu}\right|>|y|$ for any $y \in \Sigma_{\mu}$, we have

$$
\begin{aligned}
& u_{\mu}(x)-u(x) \\
& \leq \int_{\Sigma_{\mu}}\left(\left[\frac{1}{|x-y|^{\lambda}}-\frac{1}{\left|x^{\mu}-y\right|^{\lambda}}\right] \frac{1}{\left|y^{\mu}\right|^{\beta}}\right)\left(v_{\mu}^{p} u_{\mu}^{q} w_{\mu}^{r}(y)-v^{p} u^{q} w^{r}\right) d y \\
& \leq \int_{\Sigma_{\mu}^{\nu}}\left(\left[\frac{1}{|x-y|^{\lambda}}-\frac{1}{\left|x^{\mu}-y\right|^{\lambda}}\right] \frac{1}{\left|y^{\mu}\right|^{\beta}}\right)\left(\left[v_{\mu}^{p}-v^{p}\right] u_{\mu}^{q} w_{\mu}^{r}(y)\right) d y \\
& \quad+\int_{\Sigma_{\mu}^{u}}\left(\left[\frac{1}{|x-y|^{\lambda}}-\frac{1}{\left|x^{\mu}-y\right|^{\lambda}}\right] \frac{1}{\left|y^{\mu}\right|^{\beta}}\right)\left(v^{p}\left[u_{\mu}^{q}-u^{q}\right] w_{\mu}^{r}(y)\right) d y
\end{aligned}
$$




$$
\begin{aligned}
& \quad+\int_{\Sigma_{\mu}^{w}}\left(\left[\frac{1}{|x-y|^{\lambda}}-\frac{1}{\left|x^{\mu}-y\right|^{\lambda}}\right] \frac{1}{\left|y^{\mu}\right|^{\beta}}\right)\left(v^{p} u^{q}\left[w_{\mu}^{r}(y)-w^{r}\right]\right) d y \\
& \triangleq A_{1,1}(x)+A_{1,2}(x)+A_{1,3}(x) .
\end{aligned}
$$

Similarly, we conclude that

$$
\begin{aligned}
& v_{\mu}(x)-v(x) \\
& \leq \int_{\Sigma_{\mu}}\left(\left[\frac{1}{|x-y|^{\lambda}}-\frac{1}{\left|x^{\mu}-y\right|^{\lambda}}\right] \frac{1}{\left|y^{\mu}\right|^{\beta}}\right)\left(v_{\mu}^{r} u_{\mu}^{p} w_{\mu}^{q}(y)-v^{r} u^{p} w^{q}\right) d y \\
& \leq \int_{\Sigma_{\mu}^{\nu}}\left(\left[\frac{1}{|x-y|^{\lambda}}-\frac{1}{\left|x^{\mu}-y\right|^{\lambda}}\right] \frac{1}{\left|y^{\mu}\right|^{\beta}}\right)\left(\left[v_{\mu}^{r}-v^{r}\right] u_{\mu}^{p} w_{\mu}^{q}(y)\right) d y \\
& \quad+\int_{\Sigma_{\mu}^{u}}\left(\left[\frac{1}{|x-y|^{\lambda}}-\frac{1}{\left|x^{\mu}-y\right|^{\lambda}}\right] \frac{1}{\left|y^{\mu}\right|^{\beta}}\right)\left(v^{r}\left[u_{\mu}^{p}-u^{p}\right] w_{\mu}^{q}(y)\right) d y \\
& \quad+\int_{\Sigma_{\mu}^{w}}\left(\left[\frac{1}{|x-y|^{\lambda}}-\frac{1}{\left|x^{\mu}-y\right|^{\lambda}}\right] \frac{1}{\left|y^{\mu}\right|^{\beta}}\right)\left(v^{r} u^{p}\left[w_{\mu}^{q}(y)-w^{q}\right]\right) d y \\
& \triangleq \\
& B_{1,1}(x)+B_{1,2}(x)+B_{1,3}(x)
\end{aligned}
$$

and

$$
\begin{aligned}
& w_{\mu}(x)-w(x) \\
& \leq \int_{\Sigma_{\mu}}\left(\left[\frac{1}{|x-y|^{\lambda}}-\frac{1}{\left|x^{\mu}-y\right|^{\lambda}}\right] \frac{1}{\left|y^{\mu}\right|^{\beta}}\right)\left(v_{\mu}^{q} u_{\mu}^{r} w_{\mu}^{p}(y)-v^{q} u^{r} w^{p}\right) d y \\
& \leq \int_{\Sigma_{\mu}^{\nu}}\left(\left[\frac{1}{|x-y|^{\lambda}}-\frac{1}{\left|x^{\mu}-y\right|^{\lambda}}\right] \frac{1}{\left|y^{\mu}\right|^{\beta}}\right)\left(\left[v_{\mu}^{q}-v^{q}\right] u_{\mu}^{r} w_{\mu}^{p}(y)\right) d y \\
& \quad+\int_{\Sigma_{\mu}^{u}}\left(\left[\frac{1}{|x-y|^{\lambda}}-\frac{1}{\left|x^{\mu}-y\right|^{\lambda}}\right] \frac{1}{\left|y^{\mu}\right|^{\beta}}\right)\left(v^{q}\left[u_{\mu}^{r}-u^{r}\right] w_{\mu}^{p}(y)\right) d y \\
& \quad+\int_{\Sigma_{\mu}^{w}}\left(\left[\frac{1}{|x-y|^{\lambda}}-\frac{1}{\left|x^{\mu}-y\right|^{\lambda}}\right] \frac{1}{\left|y^{\mu}\right|^{\beta}}\right)\left(v^{q} u^{r}\left[w_{\mu}^{p}(y)-w^{p}\right]\right) d y \\
& \triangleq C_{1,1}(x)+C_{1,2}(x)+C_{1,3}(x),
\end{aligned}
$$

where $\Sigma_{\mu}^{u}=\left\{x \in \Sigma_{\mu} \mid u(x)<u_{\mu}(x)\right\}, \Sigma_{\mu}^{v}=\left\{x \in \Sigma_{\mu} \mid v(x)<v_{\mu}(x)\right\}$ and $\Sigma_{\mu}^{w}=\left\{x \in \Sigma_{\mu} \mid w(x)<\right.$ $\left.w_{\mu}(x)\right\}$.

Since $p+q+r=(2 n-\lambda-2 \beta) / \lambda$ and $0<\beta+\lambda<n$,

$$
s \triangleq \frac{n(p+q+r-1)}{n-\lambda-\beta}=\frac{2 n}{\lambda}>\frac{n}{\lambda} .
$$

By the weighted Hardy-Littlewood-Sobolev inequality and the Hölder inequality, we conclude that

$$
\begin{aligned}
\left\|A_{1,1}(x)\right\|_{L^{s}\left(\Sigma_{\mu}^{u}\right)} & \leq C(n, \beta, \lambda)\left\|\left[v_{\mu}^{p}-v^{p}\right] u_{\mu}^{q} w_{\mu}^{r}\right\|_{L^{\frac{n s}{n+(n-\lambda-\beta) s}}\left(\Sigma_{\mu}^{v}\right)} \\
& \leq C(n, \beta, \lambda, p, q)\left\|u_{\mu}^{q} v_{\mu}^{p-1} w_{\mu}^{r}\right\|_{L^{\frac{n}{n-\lambda-\beta}\left(\Sigma_{\mu}\right)}}\left\|v_{\mu}-v\right\|_{L^{s}\left(\Sigma_{\mu}^{v}\right)} \\
& \leq C(n, \beta, \lambda, p, q)\left\|v_{\mu}\right\|_{L^{s}\left(\Sigma_{\mu}^{v}\right)}^{p-1}\left\|w_{\mu}\right\|_{L^{s}\left(\Sigma_{\mu}\right)}^{r}\left\|u_{\mu}\right\|_{L^{s}\left(\Sigma_{\mu}\right)}^{q}\left\|v_{\mu}-v\right\|_{L^{s}\left(\Sigma_{\mu}^{v}\right)} .
\end{aligned}
$$


Similarly, we have

$$
\left\|A_{1,2}(x)\right\|_{L^{s}\left(\Sigma_{\mu}^{\nu}\right)} \leq C(n, p, q, \beta, \lambda)\|v\|_{L^{s}\left(\Sigma_{\mu}\right)}^{p}\|u\|_{L^{s}\left(\Sigma_{\mu}^{\mu}\right)}^{q-1}\left\|w_{\mu}\right\|_{L^{s}\left(\Sigma_{\mu}\right)}^{r}\left\|u_{\mu}-u\right\|_{L^{s}\left(\Sigma_{\mu}^{u}\right)}
$$

and

$$
\left\|A_{1,3}(x)\right\|_{L^{s}\left(\Sigma_{\mu}^{\nu}\right)} \leq C(n, p, q, \beta, \lambda)\|v\|_{L^{s}\left(\Sigma_{\mu}\right)}^{p}\|u\|_{L^{s}\left(\Sigma_{\mu}\right)}^{q}\left\|w_{\mu}\right\|_{L^{s}\left(\Sigma_{\mu}\right)}^{r-1}\left\|w_{\mu}-w\right\|_{L^{s}\left(\Sigma_{\mu}^{w}\right)}
$$

This together with (2.5), implies that

$$
\begin{aligned}
\left\|u_{\mu}-u\right\|_{L^{s}\left(\Sigma_{\mu}^{u}\right) \leq} \leq & \left\|A_{1,1}(x)\right\|_{L^{s}\left(\Sigma_{\mu}^{u}\right)}+\left\|A_{1,2}(x)\right\|_{L^{s}\left(\Sigma_{\mu}^{u}\right)}+\left\|A_{1,3}(x)\right\|_{L^{s}\left(\Sigma_{\mu}^{u}\right)} \\
\leq & C\left\{\left\|v_{\mu}\right\|_{L^{s}\left(\Sigma_{\mu}^{\nu}\right)}^{p-1}\left\|w_{\mu}\right\|_{L^{s}\left(\Sigma_{\mu}\right)}^{r}\left\|u_{\mu}\right\|_{L^{s}\left(\Sigma_{\mu}\right)}^{q}\left\|v_{\mu}-v\right\|_{L^{s}\left(\Sigma_{\mu}^{\nu}\right)}\right. \\
& +\|v\|_{L^{s}\left(\Sigma_{\mu}\right)}^{p}\|u\|_{L^{s}\left(\Sigma_{\mu}^{u}\right)}^{q-1}\left\|w_{\mu}\right\|_{L^{s}\left(\Sigma_{\mu}\right)}^{r}\left\|u_{\mu}-u\right\|_{L^{s}\left(\Sigma_{\mu}^{u}\right)} \\
& \left.+\|v\|_{L^{s}\left(\Sigma_{\mu}\right)}^{p}\|u\|_{L^{s}\left(\Sigma_{\mu}\right)}^{q}\left\|w_{\mu}\right\|_{L^{s}\left(\Sigma_{\mu}\right)}^{r-1}\left\|w_{\mu}-w\right\|_{L^{s}\left(\Sigma_{\mu}^{w}\right)}\right\} .
\end{aligned}
$$

Since $u, v, w \in L^{s}\left(\mathbb{R}^{n}\right)$, we can choose $N>0$ large enough, such that for any $\mu \leq-N<0$,

$$
\begin{aligned}
& C(n, \beta, \lambda, p, q)\left\{\left\|v_{\mu}\right\|_{L^{s}\left(\Sigma_{\mu}^{\nu}\right)}^{p-1}\left\|w_{\mu}\right\|_{L^{s}\left(\Sigma_{\mu}\right)}^{r}\left\|u_{\mu}\right\|_{L^{s}\left(\Sigma_{\mu}\right)}^{q}+\|v\|_{L^{s}\left(\Sigma_{\mu}\right)}^{p}\|u\|_{L^{s}\left(\Sigma_{\mu}^{u}\right)}^{q-1}\left\|w_{\mu}\right\|_{L^{s}\left(\Sigma_{\mu}\right)}^{r}\right. \\
& \left.\quad+\|v\|_{L^{s}\left(\Sigma_{\mu}\right)}^{p}\|u\|_{L^{s}\left(\Sigma_{\mu}\right)}^{q}\left\|w_{\mu}\right\|_{L^{s}\left(\Sigma_{\mu}\right)}^{r-1}\right\} \leq \frac{1}{9},
\end{aligned}
$$

which, combined with (2.11), implies that

$$
\begin{aligned}
& \left\|u_{\mu}-u\right\|_{L^{s}\left(\Sigma_{\mu}^{u}\right)} \\
& \quad \leq \frac{1}{9}\left\|u_{\mu}-u\right\|_{L^{s}\left(\Sigma_{\mu}^{u}\right)}+\frac{1}{9}\left\|v_{\mu}-v\right\|_{L^{s}\left(\Sigma_{\mu}^{\nu}\right)}+\frac{1}{9}\left\|w_{\mu}-w\right\|_{L^{s}\left(\Sigma_{\mu}^{w}\right)} .
\end{aligned}
$$

With the same method, we have

$$
\begin{aligned}
& \left\|v_{\mu}-v\right\|_{L^{s}\left(\Sigma_{\mu}^{v}\right)} \\
& \quad \leq \frac{1}{9}\left\|u_{\mu}-u\right\|_{L^{s}\left(\Sigma_{\mu}^{u}\right)}+\frac{1}{9}\left\|v_{\mu}-v\right\|_{L^{s}\left(\Sigma_{\mu}^{v}\right)}+\frac{1}{9}\left\|w_{\mu}-w\right\|_{L^{s}\left(\Sigma_{\mu}^{w}\right)}
\end{aligned}
$$

and

$$
\begin{aligned}
& \left\|w_{\mu}-w\right\|_{L^{s}\left(\Sigma_{\mu}^{w}\right)} \\
& \quad \leq \frac{1}{9}\left\|u_{\mu}-u\right\|_{L^{s}\left(\Sigma_{\mu}^{u}\right)}+\frac{1}{9}\left\|v_{\mu}-v\right\|_{L^{s}\left(\Sigma_{\mu}^{\nu}\right)}+\frac{1}{9}\left\|w_{\mu}-w\right\|_{L^{s}\left(\Sigma_{\mu}^{w}\right)} .
\end{aligned}
$$

This together with (2.4), (2.5), and (2.6) leads to

$$
\left\|u_{\mu}-u\right\|_{L^{s}\left(\Sigma_{\mu}^{u}\right)}+\left\|v_{\mu}-v\right\|_{L^{s}\left(\Sigma_{\mu}^{\nu}\right)}+\left\|w_{\mu}-w\right\|_{L^{s}\left(\Sigma_{\mu}^{w}\right)}=0
$$

Therefore $\Sigma_{\mu}^{u}, \Sigma_{\mu}^{v}$ and $\Sigma_{\mu}^{w}$ must be three zero-measure sets, which completes the assertion of Step 1. 
Step 2: We will continuously move the plane $x_{1}=\mu$ to the right as long as (2.4) holds. Indeed, suppose that at $x_{1}=\mu^{0}<0$, we have, for any $x \in \Sigma_{\mu^{0}}$

$$
u(x) \geq u_{\mu^{0}}(x), \quad v(x) \geq v_{\mu^{0}}(x), \quad \text { and } \quad w(x) \geq w_{\mu^{0}}(x)
$$

but

$$
u(x) \not \equiv u_{\mu^{0}}(x) \quad \text { or } \quad v(x) \not \equiv v_{\mu^{0}}(x) \quad \text { or } \quad w(x) \not \equiv w_{\mu^{0}}(x)
$$

Next, we will show that the plane can be moved further to the right. Precisely, there exists an $\epsilon$ depending on $n, \alpha, \lambda, \beta$ and the solution $(u(x), v(x), w(x))$ itself such that for $\forall x \in \Sigma_{\mu}$, $\mu \in\left[\mu^{0}, \mu^{0}+\epsilon\right)$

$$
u(x) \geq u_{\mu}(x), \quad v(x) \geq v_{\mu}(x), \quad \text { and } \quad w(x) \geq w_{\mu}(x)
$$

Under the assumption that $v(x) \not \equiv v_{\mu^{0}}(x)$ or $w(x) \not \equiv w_{\mu^{0}}(x)$ on $\Sigma_{\mu^{0}}$, by (2.1), (2.2), (2.3), and the non-negativity of $u, v, w$, we have $u(x)>u_{\mu^{0}}(x)$ in the interior of $\Sigma_{\mu^{0}}$.

Let

$$
\begin{aligned}
& \left\{\widetilde{\Sigma}_{\mu^{0}}^{u}=\left\{x \in \Sigma_{\mu^{0}} \mid u(x) \leq u_{\mu^{0}}(x)\right\}, \widetilde{\Sigma}_{\mu^{0}}^{v}=\left\{x \in \Sigma_{\mu^{0}} \mid v(x) \leq v_{\mu^{0}}(x)\right\}\right. \\
& \left.\quad \text { and } \widetilde{\Sigma}_{\mu^{0}}^{w}=\left\{x \in \Sigma_{\mu^{0}} \mid w(x) \leq w_{\mu^{0}}(x)\right\}\right\} .
\end{aligned}
$$

From the analysis mentioned above, it is easy to check that the $\widetilde{\Sigma}_{\mu^{0}}^{u}$ is a zero measure set in $\mathbb{R}^{n}$. Similarly, we also have $m\left\{\widetilde{\Sigma}_{\mu^{0}}^{v}\right\}=0$ and $m\left\{\widetilde{\Sigma}_{\mu^{0}}^{w}\right\}=0$. This together with the integrability conditions $(u, v, w) \in L^{S}\left(\mathbb{R}^{n}\right)$ ensures that one can choose $\epsilon$ small enough such that for all $\mu \in\left[\mu^{0}, \mu^{0}+\epsilon\right)$

$$
\begin{aligned}
& C(n, \beta, \lambda, p, q)\left\{\left[\left\|v_{\mu}\right\|_{L^{s}\left(\Sigma_{\mu}^{v}\right)}^{p-1}\left\|w_{\mu}\right\|_{L^{s}\left(\Sigma_{\mu}\right)}^{r}\left\|u_{\mu}\right\|_{L^{s}\left(\Sigma_{\mu}\right)}^{q}+\|v\|_{L^{s}\left(\Sigma_{\mu}\right)}^{p}\|u\|_{L^{s}\left(\Sigma_{\mu}^{u}\right)}^{q-1}\left\|w_{\mu}\right\|_{L^{s}\left(\Sigma_{\mu}\right)}^{r}\right.\right. \\
& \left.\quad+\|v\|_{L^{s}\left(\Sigma_{\mu}\right)}^{p}\|u\|_{L^{s}\left(\Sigma_{\mu}\right)}^{q}\left\|w_{\mu}\right\|_{L^{s}\left(\Sigma_{\mu}^{w}\right)}^{r-1}\right]+\left[\left\|v_{\mu}\right\|_{L^{s}\left(\Sigma_{\mu}^{v}\right)}^{r-1}\left\|u_{\mu}\right\|_{L^{s}\left(\Sigma_{\mu}\right)}^{p}\left\|w_{\mu}\right\|_{L^{s}\left(\Sigma_{\mu}\right)}^{p}\right. \\
& \left.\quad+\|v\|_{L^{s}\left(\Sigma_{\mu}\right)}^{r}\left\|u_{\mu}\right\|_{L^{s}\left(\Sigma_{\mu}^{u}\right)}^{p-1}\left\|w_{\mu}\right\|_{L^{s}\left(\Sigma_{\mu}\right)}^{q}+\|v\|_{L^{s}\left(\Sigma_{\mu}\right)}^{r}\|u\|_{L^{s}\left(\Sigma_{\mu}\right)}^{p}\left\|w_{\mu}\right\|_{L^{s}\left(\Sigma_{\mu}\right)}^{q-1}\right] \\
& \quad+\left[\left\|v_{\mu}\right\|_{L^{s}\left(\Sigma_{\mu}^{v}\right)}^{q-1}\left\|u_{\mu}\right\|_{L^{s}\left(\Sigma_{\mu}\right)}^{r}\left\|w_{\mu}\right\|_{L^{s}\left(\Sigma_{\mu}\right)}^{p}+\|v\|_{L^{s}\left(\Sigma_{\mu}\right)}^{q}\left\|u_{\mu}\right\|_{L^{s}\left(\Sigma_{\mu}^{u}\right)}^{r-1}\left\|w_{\mu}\right\|_{L^{s}\left(\Sigma_{\mu}\right)}^{p}\right. \\
& \left.\left.\quad+\|v\|_{L^{s}\left(\Sigma_{\mu}\right)}^{q}\|u\|_{L^{s}\left(\Sigma_{\mu}\right)}^{r}\left\|w_{\mu}\right\|_{L^{s}\left(\Sigma_{\mu}^{w}\right)}^{p-1}\right]\right\} \leq \frac{1}{27} .
\end{aligned}
$$

So (2.13) and (2.14) hold. Thus we also have

$$
\left\|u_{\mu}(x)-u(x)\right\|_{L^{s}\left(\Sigma_{\mu}^{u}\right)}=\left\|v_{\mu}(x)-v(x)\right\|_{L^{s}\left(\Sigma_{\mu}^{v}\right)}=\left\|w_{\mu}(x)-w(x)\right\|_{L^{s}\left(\Sigma_{\mu}^{v}\right)}=0
$$

which implies that the measures of $\widetilde{\Sigma}_{\mu}^{u}, \widetilde{\Sigma}_{\mu}^{v}$, and $\widetilde{\Sigma}_{\mu}^{w}$ must be zero. This verifies (2.15). Finally, we show that the plane cannot stop before hitting the origin. On the contrary, if the plane stops at $x_{1}=\mu^{0}<0$, then $u(x), v(x)$, and $w(x)$ must be symmetric about the plane $x_{1}=\mu^{0}$, i.e.,

$$
u(x)=u_{\mu^{0}}(x), \quad v(x)=v_{\mu^{0}}(x), \quad \text { and } \quad w(x)=w_{\mu^{0}}(x), \quad \forall x \in \Sigma_{\mu^{0}} .
$$


On the other hand, noting that $\left|x^{\mu^{0}}\right|>|x|$ for any $x \in \Sigma_{\mu^{0}}$, we have

$$
\begin{aligned}
& u(x)-u_{\mu^{0}}(x) \\
& =\int_{\mathbb{R}^{n}} \frac{v^{p}(y) u^{q}(y) w^{r}(y)}{|x-y|^{\lambda}} \frac{1}{|y|^{\beta}} d y-\int_{\mathbb{R}^{n}} \frac{v^{p}(y) u^{q}(y) w^{r}(y)}{\left|x^{\mu^{0}}-y\right|^{\lambda}} \frac{1}{|y|^{\beta}} d y \\
& \geq \int_{\mathbb{R}^{n}}\left[\frac{1}{|x-y|^{\lambda}}-\frac{1}{\left|x^{\mu^{0}}-y\right|^{\lambda}}\right] \frac{v^{p}(y) u^{q}(y) w^{r}(y)}{|y|^{\beta}} d y \\
& =\int_{\Sigma_{\mu^{0}}}\left[\frac{1}{|x-y|^{\lambda}}-\frac{1}{\left|x^{\mu^{0}}-y\right|^{\lambda}}\right]\left(\frac{v^{p} u^{q} w^{r}(y)}{|y|^{\beta}}-\frac{v_{{ }^{0}}^{p} u_{\mu^{0}}^{q} w_{\mu^{0}}^{r}(y)}{\left|y^{\mu^{0}}\right|^{\beta}}\right) d y \\
& >\int_{\Sigma_{\mu^{0}}}\left[\frac{1}{|x-y|^{\lambda}}-\frac{1}{\left|x^{\mu^{0}}-y\right|^{\lambda}}\right]\left\{\frac{v^{p} u^{q} w^{r}(y)-v_{\mu^{0}}^{p}(y) u_{\mu^{0}}^{q} w_{\mu}^{r}(y)}{|y|^{\beta}}\right\} d y \\
& =0,
\end{aligned}
$$

which obviously contradicts with (2.17). Since the direction is arbitrary, we derive that $u$ and $v$ are radially symmetric about the origin and decreasing. This completes the proof of $\mathrm{R}_{1}$.

\section{Integrability}

In this section, we will apply the regularity lifting lemma to obtain the integrable intervals of the solutions of system (1.1). On the other hand, a new skill is adapted to get the uniformly bound of positive solutions. Here, for completeness, we first of all give the regularity lifting lemma as follows.

Lemma 3.1 (cf. [13]) Let $\mathcal{X}$ and $\mathcal{Y}$ be both Banach spaces with norm $\|\cdot\| \mathcal{X}$ and $\|\cdot\| \mathcal{Y}$, respectively. The subspace of $\mathcal{X}$ and $\mathcal{Y}, \mathcal{Z}=\mathcal{X} \cap \mathcal{Y}$ is endowed with a new norm by

$$
\|\cdot\|_{\mathcal{Z}}=\sqrt[p]{\|\cdot\|_{\mathcal{X}}^{p}+\|\cdot\|_{\mathcal{Y}}^{p}}, \quad p \in[1, \infty] .
$$

Suppose that $\mathcal{T}$ is a contraction map from a Banach space $\mathcal{X}$ into itself and from a Banach space $\mathcal{Y}$ into itself. If $\in \mathcal{X}$ and there exists a function $g \in \mathcal{Z}=\mathcal{X} \cap \mathcal{Y}$ such that $f=\mathcal{T} f+g$, then $f$ also belongs to $\mathcal{Z}$.

Theorem 3.2 For $s=n(p+q+r-1) /(n-\bar{\lambda})$, let $(u(x), v(x), w(x)) \in L^{s}\left(\mathbb{R}^{n}\right) \times L^{s}\left(\mathbb{R}^{n}\right) \times L^{s}\left(\mathbb{R}^{n}\right)$ be a positive solutions of (1.1). Then

$$
u(x), v(x), w(x) \in L^{\tau}\left(\mathbb{R}^{n}\right), \quad \forall \tau \in\left(\frac{n}{\lambda}, \infty\right] .
$$

Proof For every fixed real number $A>0$, set

$$
f_{A}(x) \triangleq \begin{cases}f(x), & \text { if } f(x) \geq A, \text { or }|x| \geq A \\ 0, & \text { otherwise }\end{cases}
$$

and

$$
f(x) \triangleq v(x)+u(x)+w(x) .
$$


By (1.1), we have

$$
\begin{aligned}
f(x) & =u(x)+v(x)+w(x) \\
& =\int_{\mathbb{R}^{n}}\left\{u^{p}(y) v^{q}(y) w^{r}(y)+u^{q}(y) v^{r}(y) w^{p}(y)+u^{r}(y) v^{p}(y) w^{q}(y)\right\} \frac{d y}{|y|^{\beta}|x-y|^{\lambda}} \\
& \leq C(p, q, r) \int_{\mathbb{R}^{n}} \frac{f^{p+q+r}(y)}{|x-y|^{\lambda}} \frac{1}{|y|^{\beta}} d y .
\end{aligned}
$$

On the other hand, denote $B(x)$ as follows:

$$
B(x) \triangleq \frac{f(x)}{\int_{\mathbb{R}^{n}} \frac{f^{p+q+r}(y)}{|x-y|^{\lambda}} \frac{1}{|y|^{\beta}} d y}, \quad \forall x \in \mathbb{R}^{n}
$$

Therefore, this, together with (3.2), implies that $B(x)$ is a bounded positive function in $\mathbb{R}^{n}$ and

$$
f(x)=B(x) \int_{\mathbb{R}^{n}} \frac{f^{p+q+r}(y)}{|x-y|^{\lambda}} \frac{1}{|y|^{\beta}} d y, \quad \forall x \in \mathbb{R}^{n} .
$$

To obtain the integrability of $f(x)$, define the following functional:

$$
M_{A}(g)(x) \triangleq B(x) \int_{\mathbb{R}^{n}} \frac{f_{A}^{p+q+r-1}(y) g(y)}{|x-y|^{\lambda}} \frac{1}{|y|^{\beta}} d y, \quad \forall x \in \mathbb{R}^{n}
$$

and

$$
R(x) \triangleq B(x) \int_{\mathbb{R}^{n}} \frac{\left(f-f_{A}\right)^{p+q+r}(y)}{|x-y|^{\lambda}} \frac{1}{|y|^{\beta}} d y, \quad \forall x \in \mathbb{R}^{n} .
$$

Obviously, by (1.1), $f(x)$ is a positive solution of the following equation:

$$
f(x)=M_{A}(f)(x)+R(x)
$$

To obtain the integrability of $f(x)$, we first of all build up a prior estimate of $M_{A}(g)(x)$ and $R(x)$. Observe that $f(x) \in L^{\frac{(p+q+r-1) n}{n-\lambda-\beta}}\left(\mathbb{R}^{n}\right)$, by the weighted Hardy-Littlewood-Sobolev inequality, we conclude that for $\forall r \in(n / \lambda,+\infty)$

$$
\begin{aligned}
\left\|M_{A}(g)(x)\right\|_{r} & \leq C(\beta, \lambda, n)\left\|f_{A}^{p+q+r-1} g\right\|_{\frac{n r}{n+(n-\lambda-\beta) r}} \\
& \leq C(\beta, \lambda, n)\left\|f_{A}\right\|_{\frac{(p+q+r-1) n}{n-\lambda-\beta}}^{p+q+r-1}\|g\|_{r} .
\end{aligned}
$$

Hence, according to the definition of $f_{A}$, we can choose a real number $A$ large enough such that

$$
\left\|f_{A}\right\|_{\frac{(p+q+r-1) n}{n-\lambda-\beta}}^{p+q+r-1} \leq \frac{1}{2}
$$

which together with (3.4) implies that $M_{A}(g)$ is a contraction mapping from $L^{r}\left(\mathbb{R}^{n}\right)$ to itself. Similarly, noting that $f(x)-f_{A}(x)$ is a bounded function with compacted set, therefore, for 
$\forall r \in(n / \lambda, \infty)$, we have

$$
\|R(x)\|_{r} \leq C\left(\|B(x)\|_{\infty}\right)\left\|\left(f-f_{A}\right)^{p+q+r}\right\|_{\frac{n r}{n+(n-\lambda-\beta) r}} .
$$

Now, we are in a position to apply Lemma 3.1 to obtain the sharp integrability of $f$. Taking $\mathcal{X}=L^{\frac{(p+q+r-1) n}{n-\lambda-\beta}}\left(\mathbb{R}^{n}\right)$ and $\mathcal{Y}=L^{r}\left(\mathbb{R}^{n}\right), r \in(n / \lambda, \infty)$, in Lemma 3.1, this together with $[(p+q+$ $r-1) n] /(n-\lambda-\beta)=2 n / \lambda>n / \lambda$ implies that

$$
f \in L^{\tau}\left(\mathbb{R}^{n}\right), \quad \tau \in\left(\frac{n}{\lambda},+\infty\right)
$$

Next, we consider the end-point case. By (3.3), it suffices to obtain the boundedness of $M(f)(x)$ and $R(x)$. Indeed, as $|x|>2 A$ and $|y|<A$, we have $|x-y|>A$ and

$$
\begin{aligned}
R(x) & =B(x) \int_{\mathbb{R}^{n}} \frac{\left(f-f_{A}\right)^{p+q+r}(y)}{|x-y|^{\lambda}} \frac{1}{|y|^{\beta}} d y \\
& \leq B(x) A^{p+q+r-\lambda} \int_{|y|<A} \frac{1}{|y|^{\beta}} d y \leq C\left(n,\|B(x)\|_{\infty}\right) A^{p+q+r+n-\lambda-\beta} .
\end{aligned}
$$

At the same time, by Hölder's inequality, we derive that for $|x| \leq 2 A$

$$
\begin{aligned}
R(x) & \leq B(x) A^{p+q+r-\lambda} \int_{|y|<A} \frac{1}{|x-y|^{\lambda}} \frac{1}{|y|^{\beta}} d y \\
& \leq\|B(x)\|_{\infty} A^{p+q+r-\lambda}\left(\int_{|y|<A}\left(\frac{1}{|y|^{\beta}}\right)^{\frac{\lambda+\beta}{\beta}} d y\right)^{\frac{\beta}{\beta+\lambda}}\left(\int_{|y-x|<3 A}\left[\frac{1}{|y-x|^{\lambda}}\right]^{\frac{\lambda+\beta}{\lambda}} d y\right)^{\frac{\lambda}{\lambda+\beta}} \\
& =C\left(\|B\|_{\infty}\right) A^{p+q+r-\beta-\lambda+n} .
\end{aligned}
$$

Next we discuss the boundedness of $M_{A}(f)(x)$. At first, we decompose $M_{A}(f)(x)$ as follows:

$$
\begin{aligned}
M_{A}(f)(x) & =B(x) \int_{\mathbb{R}^{n}} \frac{f_{A}^{p+q+r}(y)}{|x-y|^{\lambda}}|y|^{-\beta} d y \\
& =B(x)\left(\int_{|y|<A}+\int_{|y| \geq A}\right) \int_{\mathbb{R}^{n}} \frac{f_{A}^{p+q+r}(y)}{|x-y|^{\lambda}} \frac{1}{|y|^{\beta}} d y \\
& \triangleq M_{A, 1}(f)(x)+M_{A, 2}(f)(x) .
\end{aligned}
$$

Note that

$$
\frac{n(p+q+r-1)}{(n-\beta-\lambda)(p+q+r)}=\frac{2 n}{2 n-\lambda-2 \beta} \quad \text { and } \quad n-\frac{2 n \beta}{\lambda+2 \beta}>0 .
$$

Therefore when $|x|>2 A$ and $|y|<A$, it is easy to verify that $|x-y|>A$ and

$$
\begin{aligned}
M_{A, 1}(f)(x) & \leq \int_{|y|<A} \frac{f_{A}^{p+q+r}(y)}{|x-y|^{\lambda}} \frac{1}{|y|^{\beta}} d y \\
& \leq \frac{1}{A^{\lambda}}\left\{\int_{|y|<A} f_{A}^{\frac{n(p+q+r-1)}{n-\lambda-\beta}}(y) d y\right\}^{\frac{(p+q+r)(n-\lambda-\beta)}{n(p+q+r-1)}}\left(\int_{|y|<A}|y|^{-\beta \times \frac{2 n}{\lambda+2 \beta}} d y\right)^{\frac{\lambda+2 \beta}{2 n}} \\
& <\infty
\end{aligned}
$$


Similarly, for $|x|<2 A$, since $\beta+\lambda<n$, there exists a positive real number $\varepsilon$ such that

$$
0<\varepsilon<\frac{n-\beta-\lambda}{8 n} \text {. }
$$

Let $1 / s_{1}=\beta / n+\varepsilon, 1 / s_{2}=\lambda / n+\varepsilon$ and $1 / s_{3}=(n-\lambda-\beta) / 4 n$, then, together with Hölder's inequality, we have

$$
\begin{aligned}
M_{A, 1}(f)(x) \leq & C(A, n)\left(\int_{|y|<A}|y|^{-\beta s_{1}} d y\right)^{\frac{1}{s_{1}}} \\
& \times\left(\int_{|x-y|<3 A}|x-y|^{-\lambda s_{2}} d y\right)^{\frac{1}{s_{2}}} \cdot\left\{\int_{|y|<A} f_{A}^{(p+q+r) s_{3}}(y) d y\right\}^{\frac{1}{s_{3}}} .
\end{aligned}
$$

Next, we turn our attention to $M_{A, 2}(f)(x)$. After a basic calculation, we derive

$$
\begin{aligned}
M_{A, 2}(f)(x) & \leq C \int_{|y| \geq A} \frac{f_{A}^{p+q+r}(y)}{|x-y|^{\lambda}} \frac{1}{|y|^{\beta}} d y \\
& \leq \int_{\left(\mathbb{R}^{n} \backslash B_{A}(0)\right) \cap\left(\mathbb{R}^{n} \backslash B_{A}(x)\right)} \frac{f_{A}^{p+q+r}(y)}{|x-y|^{\lambda}} \frac{1}{|y|^{\beta}} d y+\frac{1}{A^{\beta}} \int_{|x-y| \leq A} \frac{f_{A}^{p+q+r}(y)}{|x-y|^{\lambda}} d y \\
& \triangleq M_{A, 2,1}(f)(x)+M_{A, 2,1}(f)(x) .
\end{aligned}
$$

Take the parameters $r_{1}, s_{1}, \tau_{1}$ as follows:

$$
\frac{1}{r_{1}}=\frac{2 \lambda+\beta}{2 n}, \quad \frac{1}{s_{1}}=\frac{\beta}{2 n}, \quad \frac{1}{\tau_{1}}=\frac{2 n-2 \lambda-2 \beta}{2 n} .
$$

By Hölder's inequality, we conclude that

$$
\begin{aligned}
M_{A, 2,1}(f)(x)= & \frac{1}{A^{\beta}} \int_{|x-y| \leq A} \frac{f_{A}^{p+q+r}(y)}{|x-y|^{\lambda}} d y \\
\leq & C(n, A)\left(\int_{|x-y|<A} f_{A}^{(p+q+r) s_{1}}(y) d y\right)^{\frac{1}{s_{1}}} \\
& \times\left(\int_{|x-y|<A}|x-y|^{-\lambda r_{1}} d y\right)^{\frac{1}{r_{1}}} A^{\frac{n}{\tau_{1}}} .
\end{aligned}
$$

Noting that $\beta<n$, then there exists a positive number $\varepsilon$ small enough satisfying

$$
\frac{n-\beta-\lambda}{n}+\varepsilon<\frac{2 n-2 \beta-\lambda}{n} \text { and } \varepsilon<\frac{\beta+\lambda}{n} .
$$

Set

$$
\frac{1}{s^{\prime}}=\frac{\beta+\lambda}{n}-\varepsilon \text { and } \quad \frac{1}{s}=\frac{n-(\beta+\lambda)}{n}+\varepsilon .
$$

Then

$$
\frac{1}{s(p+q+r)}=\left[\frac{n-(\beta+\lambda)}{n}+\varepsilon\right] \times \frac{\lambda}{2 n-2 \beta-\lambda}<\frac{\lambda}{n},
$$


and with Hölder's inequality

$$
\begin{aligned}
M_{A, 2,1}(f)(x)= & \int_{\left(\mathbb{R}^{n} \backslash B_{A}(0)\right) \cap\left(\mathbb{R}^{n} \backslash B_{A}(x)\right)} \frac{f_{A}^{p+q+r}(y)}{|x-y|^{\lambda}} \frac{1}{|y|^{\beta}} d y \\
\leq & \int_{\mathbb{R}^{n} \backslash B_{A}(x)} \frac{f_{A}^{p+q+r}(y)}{|x-y|^{\lambda+\beta}} d y+\int_{\mathbb{R}^{n} \backslash B_{A}(0)} \frac{f_{A}^{p+q+r}(y)}{|y|^{\beta+\lambda}} d y \\
\leq & \left(\int_{\mathbb{R}^{n} \backslash B_{A}(0)} f_{A}^{(p+q+r) s}(y)\right)^{\frac{1}{s}}\left(\int_{\mathbb{R}^{n} \backslash B_{A}(0)} \frac{1}{|y|^{(\beta+\lambda) s^{\prime}}} d y\right)^{\frac{1}{s^{\prime}}} \\
& +\left(\int_{\mathbb{R}^{n} \backslash B_{A}(x)} f_{A}^{(p+q+r) s}(y)\right)^{\frac{1}{s}}\left(\int_{\mathbb{R}^{n} \backslash B_{A}(x)} \frac{1}{|x-y|^{(\beta+\lambda) s^{\prime}}} d y\right)^{\frac{1}{s^{\prime}}} .
\end{aligned}
$$

Obviously, (3.1) directly follows from (3.5) and (3.6)-(3.12). This completes the proof of Theorem 3.2.

Remark 3.3 The integrability of $(u(x), v(x), w(x))$ in (1.1) is optimal. Indeed, as $|x| \geq 2$ and $|y| \leq 1$, we have

$$
\begin{aligned}
u(x) & \geq \int_{|y| \leq 1} \frac{v^{p}(y) u^{q}(y) w^{r}(y)}{(|x|+|y|)^{\lambda}} \frac{1}{|y|^{\beta}} d y \\
& \geq C(\lambda, p, q, r)|x|^{-\lambda} \int_{|y|<1}|y|^{-\beta} d y
\end{aligned}
$$

and

$$
\int_{\mathbb{R}^{n}}\|u(x)\|_{\tau} \geq C(\lambda, \beta, p, q, r)\left(\int_{|y| \geq 2}|y|^{-\lambda \tau} d y\right)^{1 / \tau}=\infty, \quad \forall \tau \leq n / \lambda
$$

Remark 3.4 Note that $M_{A}(g)(x)$ is not a contraction mapping from $L^{\infty}\left(\mathbb{R}^{n}\right)$ to $L^{\infty}\left(\mathbb{R}^{n}\right)$, therefore the regularity lifting lemma is unavailable and we have to look for a new way to obtain the bound estimate.

\section{Asymptotic behavior}

In this section, we show $\mathrm{R}_{3}$, which implies the sharp decay rates of $u$ and $v$ at infinity. The proof is made up of two propositions.

Proposition 4.1 The following improper integrals are convergent:

$$
\begin{aligned}
& u_{\infty} \triangleq \int_{\mathbb{R}^{n}} v^{p}(y) u^{q}(y) w^{r}(y)|y|^{-\beta} d y, \\
& v_{\infty} \triangleq \int_{\mathbb{R}^{n}} v^{r}(y) u^{p}(y) w^{q}(y)|y|^{-\beta} d y, \\
& w_{\infty} \triangleq \int_{\mathbb{R}^{n}} v^{q}(y) u^{r}(y) w^{p}(y)|y|^{-\beta} d y .
\end{aligned}
$$

Proof Note that

$$
\frac{n(p+q+r-1)}{(n-\beta-\lambda)(p+q+r)}=\frac{2 n}{2 n-\lambda-2 \beta} \quad \text { and } \quad n-\frac{2 n \beta}{\lambda+2 \beta}>0 .
$$


This, together with the integrability of $f(x)$ and Hölder's equality, implies that

$$
\begin{aligned}
\mathbb{S}_{\infty} & \triangleq \int_{\mathbb{R}^{n}}\left\{v^{p}(y) u^{q}(y) w^{r}(y)+v^{r}(y) u^{p}(y) w^{q}(y)+v^{q}(y) u^{r}(y) w^{p}(y)\right\}|y|^{-\beta} d y \\
& \leq C(p, q, r)\left(\int_{|y|<A}+\int_{|y| \geq A}\right) f^{p+r+q}(y)|y|^{-\beta} d y \\
& \leq C(p, q, r, \beta, \lambda)\left\{A^{n-\beta}\|f\|_{\infty}^{p+q+r}+\|f\|_{\frac{n+(p+q+r-1)}{n-\lambda-\beta}}^{p+q+r} A^{\frac{\lambda}{2}}\right\},
\end{aligned}
$$

where $A>0$ is a real number large enough. This completes the proof of Proposition 4.1.

\section{Proposition 4.2}

$$
\begin{aligned}
& \lim _{|x| \rightarrow \infty}\left[|x|^{\lambda} u(x)\right]=\int_{\mathbb{R}^{n}} v^{p}(y) u^{q}(y) w^{r}(y)|y|^{-\beta} d y, \\
& \lim _{|x| \rightarrow \infty}\left[|x|^{\lambda} v(x)\right]=\int_{\mathbb{R}^{n}} v^{r}(y) u^{p}(y) w^{q}(y)|y|^{-\beta} d y, \\
& \lim _{|x| \rightarrow \infty}\left[|x|^{\lambda} w(x)\right]=\int_{\mathbb{R}^{n}} v^{q}(y) u^{r}(y) w^{p}(y)|y|^{-\beta} d y .
\end{aligned}
$$

Proof Define first of all $\mathscr{A}_{1}(x), \mathcal{A}_{2}(x), \mathscr{A}_{3}(x)$, respectively, as follows:

$$
\begin{aligned}
& \mathcal{A}_{1}(x) \triangleq \int_{B_{R}(0)} \frac{\nu^{p}(y) u^{q}(y) w^{r}(y)}{|y|^{\beta}} \frac{|x|^{\lambda}}{|x-y|^{\lambda}} d y, \\
& \mathcal{A}_{2}(x) \triangleq \int_{\left(\mathbb{R}^{n} \backslash B_{R}(0)\right) \backslash B_{\frac{|x|}{2}}(x)} \frac{\nu^{p}(y) u^{q}(y) w^{r}(y)}{|y|^{\beta}} \frac{|x|^{\lambda}}{|x-y|^{\lambda}} d y, \\
& \mathcal{A}_{3}(x) \triangleq \int_{B_{\frac{|x|}{2}}(x)} \frac{\nu^{p}(y) u^{q}(y) w^{r}(y)}{|y|^{\beta}} \frac{|x|^{\lambda}}{|x-y|^{\lambda}} d y .
\end{aligned}
$$

Observe that when $y \in B_{R}(0)$ and $|x| \geq 2 R$, by (4.1), we conclude that

$$
\frac{v^{p}(y) u^{q}(y) w^{r}(y)}{|y|^{\beta}}\left|\frac{|x|^{\lambda}}{|x-y|^{\lambda}}-1\right| \leq C(\lambda) \frac{\nu^{p}(y) u^{q}(y) w^{r}(y)}{|y|^{\beta}} \in L^{1}\left(\mathbb{R}^{n}\right)
$$

and with Lebesgue's dominated convergence theorem,

$$
\lim _{|x| \rightarrow \infty}\left\{\int_{B_{R}(0)} \frac{\nu^{p}(y) u^{q}(y) w^{r}(y)}{|y|^{\beta}}\left(\frac{|x|^{\lambda}}{|x-y|^{\lambda}}-1\right) d y\right\}=0,
$$

which means that

$$
\lim _{R \rightarrow \infty} \lim _{|x| \rightarrow \infty} \mathcal{A}_{1}(x)=\int_{\mathbb{R}^{n}} \frac{v^{p}(y) u^{q}(y) w^{r}(y)}{|y|^{\beta}}=u_{\infty} .
$$

To obtain (4.4), it suffices to prove $\lim _{|x| \rightarrow \infty} \mathcal{A}_{2}(x)=0$ and $\lim _{|x| \rightarrow \infty} \mathcal{A}_{3}(x)=0$. Noting that $y \in\left(\mathbb{R}^{n} \backslash B_{R}(0)\right) \backslash B_{\frac{|x|}{2}}(x)$ and $|x|>2 R$, by (4.1), we have

$$
\left|\mathcal{A}_{2}(x)\right| \leq C(\lambda) \int_{\left(\mathbb{R}^{n} \backslash B_{R}(0)\right)} \frac{v^{p}(y) u^{q}(y) w^{r}(y)}{|y|^{\beta}} d y \rightarrow 0, \quad \text { as } R \rightarrow 0 .
$$


Now, we turn to $\mathcal{A}_{3}(x)$. By Theorem 3.2 and the result of $\mathrm{R}_{1}$ in Theorem 1.1, we conclude that, for any $\tau \in(n / \lambda, \infty)$,

$$
f^{\tau}(|x|) \int_{|x| / 2<y<|x|} d y \leq \int_{|x| / 2<y<|x|} f^{\tau}(y) d y=\|f\|_{\tau}^{\tau},
$$

which implies that $f(|x|)|x|^{\frac{n}{\tau}} \leq C$ for $|x| \geq 2 R$ and

$$
\begin{aligned}
\left|\mathcal{A}_{3}(x)\right| & \leq \int_{B_{\frac{|x|}{2}}(x)} \frac{f^{p+q+r}(y)}{|y|^{\beta}} \frac{|x|^{\lambda}}{|x-y|^{\lambda}} d y \\
& \leq C(\lambda)|x|^{\lambda-\beta} f^{p+q+r}\left(\frac{|x|}{2}\right) \int_{B_{\frac{|x|}{2}}(x)}|x-y|^{-\lambda} d y \\
& =C(\lambda, n, \beta)|x|^{n-\beta-\frac{(p+q+r) n}{\tau}} .
\end{aligned}
$$

On the other hand, noting that

$$
n-\beta-[(p+q+r) n] \frac{\lambda}{n}=\lambda+\beta-n<0,
$$

and taking $1 / \tau=\lambda / n-\varepsilon$ with $\varepsilon$ being a small positive number, we derive that

$$
n-\beta-[(p+q+r) n] \frac{1}{\tau}<0
$$

and

$$
\lim _{|x| \rightarrow \infty}\left|\mathcal{A}_{3}(x)\right|=0
$$

Then, combining with (4.10) and (4.11), we obtain (4.4). Similarly, we can get (4.5) and (4.5). Therefore, this completes the proof of Proposition 4.2.

\section{Competing interests}

The authors declare they have no competing interests.

\section{Authors' contributions}

All authors contributed equally to the writing of this paper. All authors read and approved the final manuscript.

\section{Author details}

${ }^{1}$ School of Mathematics and Physics, North China Electric Power University, Beijing, 102206, China. ${ }^{2}$ School of Applied Mathematics, Xiamen University of Technology, Xiamen, 361024, China. ${ }^{3}$ College of Sciences, Hunan Agriculture University, Hunan, 410128, China.

\section{Acknowledgements}

The first author was partially supported by the National Natural Science Foundation of China (Grant: U1430103), by the fund for Beijing Higher Education Young Elite Teacher Project (Grant: YETP0724) and by the Fundamental Research Funds for the Central Universities (Grant: 13MS35). The third author was partially supported by the National Natural Science Foundation of China (Grant: 11126148).

Received: 18 August 2015 Accepted: 1 December 2015 Published online: 04 January 2016

\section{References}

1. Lu, G, Zhu, J: Symmetry and regularity of extremals of an integral equation related to the Hardy-Sobolev inequality. Calc. Var. Partial Differ. Equ. 42, 563-577 (2011)

2. Lei, Y: Asymptotic properties of positive solutions of the Hardy-Sobolev type equations. J. Differ. Equ. 254, 1774-1799 (2013) 
3. Li, C, Ma, L: Uniqueness of positive bound states to Schrödinger systems with critical exponents. SIAM J. Math. Anal. 40(3), 1049-1057 (2008)

4. Zhao, Y, Lei, Y: Asymptotic behavior of positive solutions of a nonlinear integral system. Nonlinear Anal. 75, 1989-1999 (2012)

5. Jin, C, Li, C: Symmetry of solutions to some systems of integral equations. Proc. Am. Math. Soc. 134, 1661-1670 (2006)

6. Lei, Y, Li, C, Ma, C: Asymptotic radial symmetry and growth estimates of positive solutions to weighted Hardy-Littlewood-Sobolev system of integral equations. Calc. Var. Partial Differ. Equ. 45, 43-61 (2012)

7. Lei, Y, Ma, C: Asymptotic behavior for solutions of some integral equations. Commun. Pure Appl. Anal. 10, 193-207 (2011)

8. Li, C, Lim, J: The singularity analysis of solutions to some integral equations. Commun. Pure Appl. Anal. 6, 453-464 (2007)

9. Jin, C, Li, C: Qualitative analysis of some systems of equations. Calc. Var. Partial Differ. Equ. 26, 447-457 (2006)

10. $\mathrm{Xu}, J, \mathrm{Wu}, \mathrm{H}, \mathrm{Tan}, \mathrm{Z}$ : Radial symmetry and asymptotic behaviors of positive solutions for certain nonlinear integral equations. J. Math. Anal. Appl. 427, 307-319 (2015)

11. Li, YY: Remark on some conformally invariant integral equations: the method of moving spheres. J. Eur. Math. Soc. 6 , 153-180 (2004)

12. Chen, W, Li, C, Ou, B: Classification of solutions for an integral equation. Commun. Pure Appl. Math. 54, 330-343 (2006)

13. Chen, W, Li, C: Methods on Nonlinear Elliptic Equations. AlMS Ser. Differ. Equ. Dyn. Syst., vol. 4 (2010)

\section{Submit your manuscript to a SpringerOpen ${ }^{\circ}$ journal and benefit from:}

- Convenient online submission

Rigorous peer review

- Immediate publication on acceptance

- Open access: articles freely available online

- High visibility within the field

- Retaining the copyright to your article 